\section{Removal of locking plates: new implant, new challenges and new solutions}

\author{
Bhavuk Garg, Tarun Goyal, Vijay Kumar, \\ Rajesh Malhotra, Prakash P. Kotwal \\ Department of Orthopaedics, \\ All India Institute of Medical Sciences, \\ New Delhi, India
}

\section{Abstract}

Removal of locking plates in many ways poses novel challenges compared to conventional plates. None of the techniques described for the removal of locking plates are adequate for all situations. We report our experience of 27 patients from whom a total of 33 locking plates were removed. We also describe a novel technique for the removal of locking plates which in our experience could be used in most of these patients because it is appropriate for all situations and, from a technical point of view, is easy to use. Our new technique consists of removing the problematic locking screw by cutting the plate on both sides of the screw hole and using the screw head-plate hole unit for removal. We analyzed all these patients for the location of the plate, number of locking screws, time of implant removal since the initial surgery, reason for removal of the plate, nature of the difficulties encountered during surgery, and any perioperative complications. A total of 43 (17.34\%) screws were difficult to remove. Twenty screws were found to be stripped, 15 were jammed and 8 were broken. Fourteen of the 20 stripped screws and all 15 jammed screws were removed using our technique. We found this technique of locking plate removal to be very versatile and useful in most of the cases in which removal was difficult. At the same time, it also causes less damage to the bone compared to other techniques.

\section{Introduction}

A locking compression plate combines the advantages of locking screws with the compression provided by the conventional plates. There has been a steep increase in the use of locking plates in the last decade, particularly in osteoporotic, comminuted fractures, periarticular fractures and with the use of minimally invasive techniques of fracture fixation. Over time, this has led to an increase in cases in which surgeons have been called upon to remove the locking plates.

Removal of locking plates in many ways poses novel challenges compared to conventional plates. Several authors have reported various difficulties they have encountered in the removal of these implants. ${ }^{1-7} \mathrm{~A}$ few novel methods have been described for the removal of the difficult hardware. But none of them seem to be foolproof and adequate for all situations. We report our experience of 27 patients from whom a total of 33 locking plates were removed. We also describe a novel technique for the removal of locking plates which in our view can be used in most of these patients because it is appropriate for all situations and, from a technical point of view, is easy to use.

\section{Materials and Methods}

A total of 27 patients (mean age 44.5 years; range 23-62 years; 18 male and 9 female) who underwent surgery for locking plate removal between January 2006 and June 2010 were included in this prospective study. A total of 33 locking plates were removed. All these patients were studied for the location of the plate, number of locking screws, time of implant removal since the initial surgery, reason for removal of the plate, nature of the difficulties encountered during surgery and any perioperative complications. X-rays were studied to evaluate the condition of the parent bone for evidence of union, signs of infection and broken hardware or bony overgrowth over the plate.

Long-term function and patient follow up after implant removal were not among the objectives of this study which only examined perioperative difficulties in implant removal.

We initially tried to remove the screws according to the routine method with a screwdriver. The screws which could not be removed with the screwdriver were assessed for any evidence of stripping. These were generally those screws in which the screwdriver could not grip the head of the screw. An attempt was made to remove these screws with the conical extraction bolt. The jammed screws were removed with the novel surgical technique described here.

\section{Surgical technique}

The plate is cut on both sides of the screw hole with a high-speed metal cutting saw leaving the hole intact (Figure 1). Special care is taken that the full thickness of the plate is not cut, as this might damage the bone cortex with the high-speed cutting saw. The plate is weakened with the saw and the remaining part is cut with a metal cutter as shown in Figure 2. This creates an independent unit consisting of the screw locked in the locking hole of the plate. This provides a larger extraosseous rectangular piece of metal to hold as compared to the smaller, round screw head. This is gripped
Correspondence: Tarun Goyal, 287 Heaton Road, Newcastle Upon Tyne, NE6 5QD, UK

Key words: locking plates, jammed screws, implant removal.

Received for publication: 7 July 2011. Accepted for publication: 19 September 2011.

This work is licensed under a Creative Commons Attribution NonCommercial 3.0 License (CC BYNC 3.0).

(C) Copyright B. Garg et al., 2011

Licensee PAGEPress, Italy

Surgical Techniques Development 2011; 1:e25

doi:10.4081/std.2011.e25

with pliers and rotated to extract the screw (Figure 3). Continuous lavage irrigation was maintained to keep local temperature low and wash out the metal debris.

\section{Results}

A total of 33 locking plates (160 locking screws, 88 conventional screws; total 248 screws) were removed. The anatomical distribution of the plates is shown in Table 1.

Mean time between the placement of implants and their removal was 2.3 years. The causes for implant removal are shown in Table 2. All these locking plates were titanium implants (AO, Synthes). The standard screwdriver provided with the respective instrumentation was used to remove 205 screws (including 88 conventional screws). These were removed easily. A total of 43 (17.34\%) screws were difficult to remove. Twenty screws were found to be stripped, 15 were jammed and 8 were broken. All these screws were self tapping screws. There were no self drilling screws. Nineteen screws were in the metaphyseal region and 14 were in the diaphyseal region.

The 8 broken screws were removed using the hollow reamer. We were able to remove only 6 of the 20 stripped screws using the conical extraction bolts. Despite two attempts with the conical extraction bolt, the remaining 14 screws could not be removed and our technique of cutting the plate before extraction was required for successful removal. All the 15 jammed screws were also removed using our technique. On no occasion were we unable to remove the screw using our technique of removal.

The original fracture was united in 23 patients. In 4 patients, preoperative X-rays showed the plate to be broken. Two of the patients developed superficial infection in the postoperative period and were treated with debridement and antibiotics. One patient, in 
whom a broken screw was removed from the shaft of ulna using a hollow reamer, later had a fracture at that site during the postoperative period and was treated with an above elbow

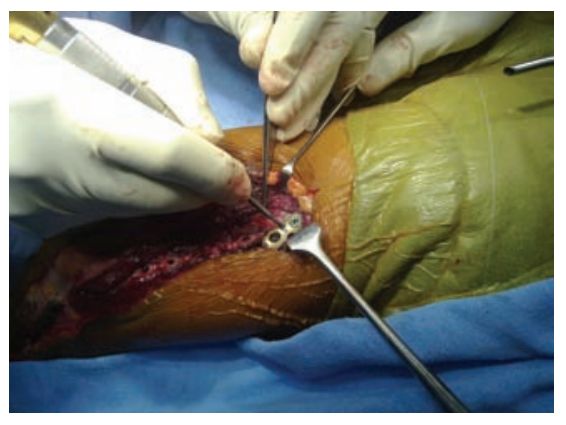

Figure 1. A high-speed metal cutting saw being used to cut the plate on both sides of the locking hole.

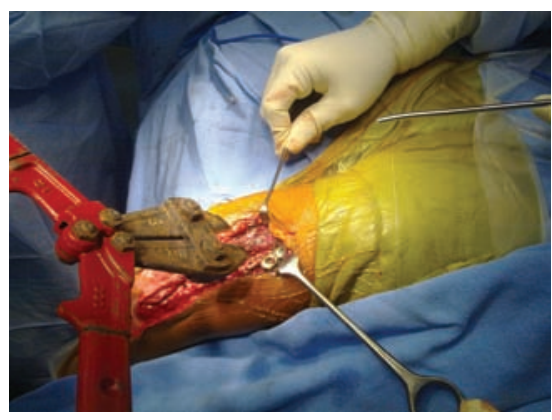

Figure 2. Cutting the plate on both sides of the hole using a metal cutter.

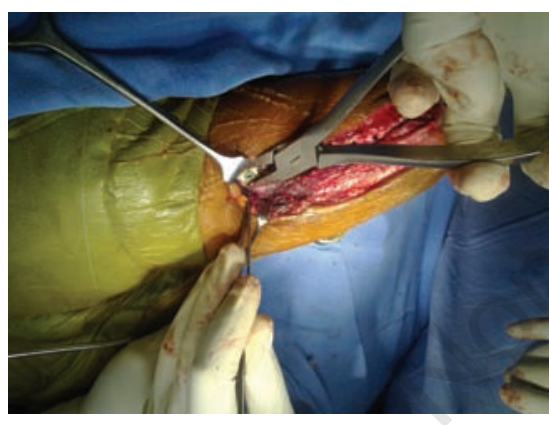

Figure 3. Using the screw head locked in the locking hole of the plate as a unit for removal of the jammed screw.

Table 1. Distribution of the locking screws found to be difficult to remove at surgery.

\begin{tabular}{lcc}
$\begin{array}{l}\text { Anatomical } \\
\text { site }\end{array}$ & $\begin{array}{c}\text { No } \\
\text { of plates }\end{array}$ & $\begin{array}{c}\text { Total no } \\
\text { of jammed } \\
\text { screws }\end{array}$ \\
Distal femur & 12 & 15 \\
Distal radius & 6 & 7 \\
\hline Humerus shaft & 5 & 6 \\
Tibia & 4 & 6 \\
\hline Proximal humerus & 3 & 5 \\
Radius shaft & 2 & 2 \\
\hline Ulna & 1 & 2 \\
Total no of plates & 33 & 43 \\
\hline
\end{tabular}

cast for six weeks. One important observation worthy of note was made of these patients: 35 of these 43 difficult screws were the second screw from the plate end. However, we are unable to explain the reason for this.

\section{Discussion}

Removal of locking plates may sometimes be very difficult, and it is mandatory that a wide range of armamentarium should be readily available. It is important to realize that, to the surprise of the surgeon, removal may be much more problematic than the initial surgery. ${ }^{8-10}$ Incision and surgical dissection during removal of the locking screws is often greater than the incision required for their placement using the minimally invasive techniques.

Some of the problems that are unique to locking plate removal include cold welding of the screw heads into the plates or cross threading between the threads on the screw head and in the plate hole. ${ }^{11,12}$ These situations may arise as a result of the use of improper techniques during insertion. ${ }^{3,6,13}$ There may be damage to the recesses on the screw head (stripping), or the screw head may be broken. Cold welding may occur in the titanium implants between the screw head and the hole of the plate, especially if the torque limiting screwdriver was not used for tightening the screw. Cross threading between the threads on the screw head and the threads on the plate may occur if the direction of insertion of the screw was not orthogonal to the direction of the hole in the plate or if the insertion sleeve was not used.

Many techniques have been described for the removal of these complicated hardware, such as conical extraction screws, hollow reamers, and carbide drill bits to cut the metal plates. Differing advice and a variety of problems have been described for the removal of jammed screws and plates, but none of the techniques is fool proof and different circumstances may necessitate the use of different techniques.

Pattison described the use of a foil between the screwdriver and screw head. ${ }^{7}$ But this is not helpful in the removal of jammed screws. An attempt can be made to use conical extraction screws but failure rates with their use is unacceptably high. ${ }^{1,45}$ They may be more useful in cases of stripped screw heads, but a larger torque may often be required in cases of cold welded or cross threaded screws. ${ }^{3,4}$ It may take more than one attempt before these extraction screws can be unthreaded. ${ }^{2}$

Kumar and Dunlop described a new technique for the removal of jammed screws by making radial cuts from the edge of the plate to the screw hole. ${ }^{1}$ An osteotome is then wedged in the cut thereby increasing the diameter of the hole in the plate. This releases the screw and makes removal possible. While this technique may be helpful in cross threaded screws, chances of failure in cold welded screws appear to be high. Furthermore, this technique cannot be used for stripped screws. There is also the possibility with this technique of exposing the nearby osteoporotic bone to greater stress and increasing the risk of creating an iatrogenic fracture.

Another technique used involves breaking up the screw head and threads into fragments with a high-speed burr but leaving the shank of the screw. The plate is then removed and then the screw shank is removed with a conical extraction screw. ${ }^{6}$ But this requires drilling a large volume of metal producing large amounts of heat and metal debris.

Georgiadis et al. described a technique to remove the stripped screws by cutting the plate around the screw head to free the screw from the plate..$^{5}$ This frees the tightly engaged screws from the plates which can then be more easily removed with a conical extraction bolt. The technique of bending the plate near the jammed screw, and using it to extract the screw, increases the risk of extreme stress to the bone and of fracture. ${ }^{4}$

Our technique to remove screws is useful in many ways. It can be used for all types of failures such as those arising from stripping the screw heads, the screw jamming into the plates or cold welding. In our experience using this technique, there have been no occasions on which the screw was not removed. All the metal work, including the entire screw shaft, can be removed using this technique. There are no reports in the literature of a similar procedure for the removal of these implants. This technique also avoids the risk of high shearing stress to the bone due to bending the plate or multiple attempts at using extraction bolts. This is especially useful in osteoporotic bones

Table 2. Summary of the technique used for the removal of the difficult locking screws

\begin{tabular}{lcl} 
Mode of screw removal & Number & Comments \\
Hollow reamer & 8 & Used for screws with broken heads \\
Conical extraction bolt & 6 & Used for stripped ( $\mathrm{n}=8)$ \\
\hline $\begin{array}{l}\text { Cutting the plate using our novel } \\
\text { technique }\end{array}$ & 29 & $\begin{array}{l}\text { Used for screws which could not be } \\
\text { extracted with the conical extraction bolt }\end{array}$ \\
$\begin{array}{l}\text { Removal with a standard screw driver } \\
\text { Ren }\end{array}$ & 205 &
\end{tabular}


where cutting the plates at two ends and screw extraction interferes very little with the bone. One disadvantage with this technique is that high-speed burrs and discs create a lot of local metal debris with high temperatures, and continuous lavage irrigation must be maintained. In theory, one factor that may lead to difficulties in extraction using this technique is bony overgrowth over the screws. This is especially important with titanium screws. This will require stripping off the bone from the screw to facilitate its removal. Another problem may arise when two adjacent screws have to be removed. This is easier in low profile plates where the high-speed metal saw will produce some gap between the plate fragments. This is technically more difficult when dealing with broader plates. A useful alternative would be to remove a fragment of plate by cutting close to the two adjacent screw holes, which are jammed. This technique is also difficult to use in less accessible sites where it would be difficult to use the large metal cutter.

\section{Conclusions}

There are, therefore, some lessons to be learned from surgery for the removal of locking implants. Firstly, prevention is the best treatment. Correct techniques should be used during screw placement, since placing locking implants is not a definitive process, and the patient may return to the same surgeon for its removal. Secondly, none of the techniques described for the removal of these implants is secure for all the patients. It is essential to have all the appropriate implant removal instruments available before starting the procedure as it may not be sufficient to adopt a single technique, and other alternative procedures may have to be considered during surgery. The surgeon should have a wide choice of options. There may be the need for greater planning than index surgery and problems need to be anticipated. Finally, our technique of implant removal has been proved to be very useful and versatile. It is not, however, a universal solution for this difficult hardware.

\section{References}

1. Kumar G, Dunlop C. Case Report: A Technique to Remove a Jammed Locking Screw from a Locking Plate. Clin Orthop Relat Res 2010;11. [Epub ahead of print]

2. Suzuki T, Smith WR, Stahel PF, et al. Technical problems and complications in the removal of the less invasive stabilization system. J Orthop Trauma 2010;24:369-73.

3. Ehlinger M, Adam P, Simon P, et al. Technical difficulties in hardware removal in titanium compression plates with locking screws. Orthop Traumatol Surg Res 2009;95:373-6.
4. Bae JH, Oh JK, Oh CW, et al. Technical difficulties of removal of locking screw after locking compression plating. Arch Orthop Trauma Surg 2009;129:91-5.

5. Georgiadis GM, Gove NK, Smith AD, et al. Removal of the less invasive stabilization system. J Orthop Trauma 2004;18:562-4.

6. Hamilton $\mathrm{P}$, Doig S, Williamson 0 . Technical difficulty of metal removal after LISS plating. Injury 2004;35:626-8.

7. Pattison G, Reynolds J, Hardy J. Salvaging a stripped drive connection when removing screws. Injury 1999;30:74-5.

8. Busam ML, Esther RJ, Obremskey WT. Hardware removal: indications and expectations. J Am Acad Orthop Surg 2006;14: 113-20.

9. Richards RH, Palmer JD, Clarke NM. Observations on removal of metal implants. Injury 1992;23:25-8.

10. Sanderson PL, Ryan W, Turner PG. Complications of metalwork removal. Injury 1992;23:29-30.

11. Hak DJ, McElvany M. Removal of broken hardware. J Am Acad Orthop Surg. 2008;16:113-20.

12. Phisitkul P, McKinley TO, Nepola JV, et al. Complications of locking plate fixation in complex proximal tibia injuries. J Orthop Trauma 2007;21:83-91.

13. Stoffel K, Dieter U, Stachowiak G, et al. How can stability in locked internal fixators be controlled? Injury 2003;34:11-9. 